\title{
Nano-Fluids in Heat Transfer: Research versus Applications
}

\author{
Andrzej W. Pacek \\ School of Chemical Engineering, University of Birmingham \\ a.w.pacek@bham.ac.uk
}

Term nano-fluids was coined by Easteman and Choi in 1995 to describe diluted suspensions of solid nano-particles in water which, as they claimed, had unusually high conductivity and were perceived to be excellent heat transfer mediums. Whilst the claim made in the 1995 paper did not have much impact (according to Science Direct 2 papers on this topic in the next 5 years), once nano-fluids were patented in US in 2001 the interest in nano-fluids and their applications in heat transfer grew exponentially and between 2001 and 2005 nearly 200 papers were published. This trend in research still continues despite the fact that, to my knowledge, there is still no commercial application of nano-fluids in the heat transfer equipment.

In this paper I will focus on the heat transfer between nano-fluids and solid surfaces commonly described by the heat transfer coefficient. I will discuss experimental literature results and compere those with recent results obtained in EU sponsored project (NANOHEX) where the thermal conductivity and the heat transfer coefficients in the range of nano-fluids and flow geometries were measured in three leading European Universities following a very stringent experimental procedure. Next I will present critical analysis of the theoretical attempts made by several researchers to explain unusually strong enhancement of the heat transfer coefficient. I will conclude with a discussion of the inherent technical problems the application of nano-fluids in commercial/industrial heat transfer equipment is facing. 